\title{
OTIOTOMICS
}

Revista de economía, empresa y sociedad

Trabajo final de grado

MEDIDAS PARA CONCILIAR EN EL SECTOR RETAIL

\section{Recomendaciones para la implementación de medidas de conciliación en el sector comercial detallista español}

\author{
Carolina Morales Pallarés \\ Graduada en Administración y Dirección de Empresas por la UOC. \\ Máster en Dirección de Marketing por EAE. \\ Especialista en retail marketing.
}

RESUMEN Los recursos humanos de una empresa constituyen una de sus principales ventajas competitivas. Valorar la importancia de este recurso y trabajar intensivamente para retener este talento constituirá uno de los principales retos de futuro de las empresas. El estudio de uno de los sectores más relevantes de la economía española, el sector comercial detallista, permite analizar la importancia de la gestión del capital humano a través de la motivación. En este punto, el concepto de conciliación personal, familiar y laboral toma relevancia como herramienta de gestión de personas y de retención de talento. Para facilitar la aplicación de medidas de conciliación se necesita: igualdad de género, paridad y corresponsabilidad. Los beneficios tanto para trabajadores como para empresas son múltiples, entre ellos el mínimo o nulo coste que supone la implantación de este tipo de medidas. Especialmente las nuevas tecnologías favorecen una conciliación real. Aportar conocimiento y dar difusión al concepto de conciliación personal, familiar y laboral, analizando su aplicabilidad en uno de los sectores más relevantes de la economía española, es el objetivo de este estudio.

Este artículo es un resumen del Trabajo Final de Grado ganador del mejor TFG de los Estudios de Economía y Empresa con perspectiva de género del curso 2018/19. El trabajo completo se encuentra depositado en el repositorio institucional de la $\mathrm{UOC}(\mathrm{O} 2)$, el portal que recoge, difunde y preserva las publicaciones digitales en acceso abierto de los miembros de la UOC, elaboradas en el desarrollo de sus actividades de investigación, docencia y gestión. Podéis consultar el trabajo completo en: http://hdl.handle.net/10609/110566.

PALABRAS CLAVE conciliación; medidas de conciliación; gestión del capital humano; motivación laboral; satisfacción laboral; retención del talento; mujer; comercio detallista; retail 


\title{
Recommendations for the implementation of measures for work-life balance in the Spanish retail commerce sector
}

\begin{abstract}
Human resources of a company constitute one of its main competitive advantages. Valuing the importance of this resource and working intensively in order to retain this talent will constitute one of the main challenges of the future for companies. The study of one of the most important sectors in the Spanish economy, the commercial retail sector, allows an analysis of the importance of the management of human capital through motivation. As part of this point, the concept of personal, family and work balance gains importance as a tool for human management and for the retention of talent. In order to facilitate the application of measures for balancing life, the following conditions are required: gender equality, parity and joint responsibility. The benefits for both workers and companies are many. Among them, the minimal or zero cost which the implementation of these kinds of measures involve. New technologies in particular assist a real balance. Providing knowledge about and spreading the concept of personal, family and work balance, by analysing its applicability in one of the most important sectors in the Spanish economy, is the objective of this study.
\end{abstract}

This article is a summary of the work which obtained the prize for the best Bachelor's Thesis (TFG) in Economics and Business Studies concerning gender during the 2018/19 course. The complete work is held in the UOC's institutional repository (O2), the portal which collects, disseminates and preserves the UOC members' free-access digital publications that were produced in the development of their research, teaching and management activities. You can consult the complete work at: http://hdl.handle.net/10609/110566.

KEYWORDS work-life balance; measures for work-life balance; human resource management; work motivation; work satisfaction; talent retention; woman; retail commerce; retail.

Conciliar es «formar dos o más proposiciones o doctrinas aparentemente contrarias» [20]. Concretamente, la acepción conciliar los ámbitos personal, familiar y laboral se refiere a «la participación equilibrada entre mujeres y hombres en la vida familiar y en el mercado laboral, conseguida a través de la reestructuración y reorganización de los sistemas laboral, educativo y de recursos sociales, con el objetivo de introducir la igualdad de oportunidades en el empleo, variar los roles y estereotipos tradicionales y cubrir las necesidades de atención y cuidado de personas con dependencia» [12].

\section{El sector comercial detallista español}

El comercio detallista (en inglés retail) se caracteriza por la venta de productos o servicios directamente al consumidor final a través de un canal físico o digital. En el caso de España, después de sufrir duramente los efectos de la crisis económica, desde el año 2015 muestra importantes signos de recuperación [13]. Constituido por 451.471 empresas, que representan un 5,5\% del PIB estatal y un 20\% del total de empleo a nivel nacional, se pone de relieve la importancia del sector en la economía española, convirtiéndose en uno de sus motores principales [4] [13] [14]. 
Según la Confederación Española de Comercio [4], en la actualidad este sector proporciona puestos de trabajo a más de 3 millones de personas, de estos, casi 2 millones pertenecen al ámbito detallista (1.331.177,50 bajo el régimen general y 524.295,90 bajo el régimen de autónomos), este último con una alta participación femenina (de un 60\% aproximadamente).

El tamaño de la empresa define su estructura organizativa, siendo mayoritarias en el sector las empresas de 2-9 trabajadores (40,1\%), seguidas de las de más de 250 trabajadores $(29,8 \%)$. La franja de edad de los trabajadores predominante se encuentra entre los 25-49 años, seguida de los trabajadores mayores de 50 años y, por último, los trabajadores entre 15-24 años. En cuestión de género, las mujeres tienen mayor peso entre los trabajadores de edad comprendida entre los 15 y los 49 años. Los hombres predominan entre los trabajadores mayores de 50 años [8].

A nivel contractual, destacar especialmente el uso del contrato a tiempo parcial frente a otros tipos de contrato [13]. El estudio Working conditions in the retail sector realizado por Eurofound el año 2012 [8] aporta interesantes conclusiones respecto a este punto: un 38\% de las empresas tienen contratado como mínimo a un trabajador a tiempo parcial. El perfil de este tipo de trabajador es: mujer con hijos pequeños, mujer sin hijos o mujer con hijos en edad escolar, estudiantes y jóvenes en su primer empleo, entre otros.

El sistema de venta predominante en el sector es el tradicional $(61,5 \%)$, seguido del autoservicio $(31,6 \%)$ y el comercio electrónico (2,9\%) [4] [13]. El horario habitual de los establecimientos del sector objeto de estudio es de entre las 9.00-10.00 h hasta las 20.00-22.00 h [16].

El sector comercial detallista en España ha sufrido una gran transformación en los últimos setenta años: iniciada en los años 60-70 con pequeñas tiendas de ultramarinos, con el tiempo evolucionó a nuevos conceptos como el autoservicio, el supermercado, el hipermercado (años 80 y 90) y finalmente el centro comercial. Paralelamente a esta última etapa nació el concepto de franquicia. Posteriormente, la aparición de la web 2.0 motivó la incorporación del sector en el medio digital. Los últimos años, caracterizados por una gran revolución tecnológica, han impuesto la complementariedad del establecimiento físico y el establecimiento electrónico debido a las nuevas demandas de los consumidores actuales. Ahora resulta imprescindible ofrecer una experiencia omnicanal.

\section{La empresa del sector retail}

Dependiendo de las características de cada empresa, la estructura organizativa toma un modelo u otro, según haya mayor o menor presencia de división del trabajo y si esta es más vertical o más horizontal. En concreto, la estructura organizativa de las empresas del sector comercial detallista es diferente según el producto comercializado y según el tamaño de la empresa.

A grandes rasgos, las empresas grandes, se caracterizan por una sede central donde están ubicados los diferentes departamentos (administración, recursos humanos, finanzas, diseño, compras, comercial y marketing), desde donde se gestionan las diversas tareas diarias o se marcan las directrices a seguir. Estos departamentos están liderados por un director de departamento. En dependencia de esta sede central hay una red de establecimientos repartidos por un territorio (nacional o internacional según el caso de la empresa) que están supervisados por uno/una responsable de zona (figura más comúnmente conocida dentro del sector como area manager) que coordina un cierto número de estos.

En el caso de las empresas pequeñas (con pocos o incluso un único establecimiento), no existe división departamental y la mayor parte de las tareas son realizadas por una sola persona o repartidas entre los miembros de un pequeño equipo. Generalmente, en estos casos se externalizan las tareas que no están directamente relacionadas con el objetivo de la empresa (como es el caso de la gestión administrativa o financiera).

El factor humano es un elemento clave para el éxito de cualquier empresa. Las características propias e intransferibles de los trabajadores se convierten en la principal ventaja competitiva de las organizaciones: es su capital humano. Así pues, aplicar una política de recursos humanos basada en la motivación permite retener este 
talento. Como se verá más adelante, las medidas de conciliación de la vida personal, familiar y laboral son una forma de motivación.

La gestión de los recursos humanos, por tanto, tendrá que plantearse bajo la visión de la gestión del capital humano. Así, aparece un nuevo objetivo a satisfacer, el cliente interno: los trabajadores.

\section{La conciliación personal, familiar y laboral}

El concepto conciliación personal, familiar y laboral queda definido como:

«La participación equilibrada entre mujeres y hombres en la vida familiar y en el mercado de trabajo, conseguida a través de la reestructuración y reorganización de los sistemas laboral, educativo y de recursos sociales, con el fin de introducir la igualdad de oportunidades en el empleo, variar los roles y estereotipos tradicionales, y cubrir las necesidades de atención y cuidado a personas dependientes.»

Palabras para la lgualdad del Plan Óptima [12]

Varios estudios muestran los rasgos más significativos de la situación actual de las medidas de conciliación personal, familiar y laboral en España:

Según indica el Segundo Estudio sobre la Situación de la Conciliación en España del IICP [3], las medidas de conciliación existentes en la actualidad han sido solicitadas y disfrutadas mayoritariamente por mujeres. Este hecho provoca que el concepto de conciliación acabe por relacionarse únicamente con el sexo femenino, penalizando de esta manera su desarrollo social, económico y sobre todo laboral. Igualmente pone de relieve la desigualdad social existente entre hombres y mujeres, y la falta de corresponsabilidad entre ambos sexos, en especial en el ámbito privado (familia y hogar).

De la misma manera, el Estudio sobre Conciliación y Familia realizado por la Fundación Pfizer [10] aporta los indicadores siguientes: un $47,1 \%$ de los encuestados declara que ser madre afecta a la trayectoria laboral de las mujeres. Por otro lado, un 39\% de las mujeres encuestadas ha tenido que renunciar a un puesto de trabajo o ascenso por el hecho de tener hijos o personas dependientes a su cargo frente al $12,3 \%$ de los hombres encuestados. Por último, pero no menos importante, un 53,3\% de los encuestados opina que sus empresas han discriminado en la selección de personal en favor de los hombres frente a un $11,5 \%$ que opina que la discriminación ha sido en favor de las mujeres. Se confirma, por tanto, la existencia real de una discriminación de gran parte de las empresas en favor de los hombres y en detrimento de las mujeres.

Múltiples son los beneficios de la implementación de medidas de conciliación, tanto para empresas como para trabajadores. En el primer caso, se pueden destacar: mejora de la imagen de la empresa y del clima laboral, aumento de su productividad, eficacia y competitividad, aumento del compromiso de sus trabajadores y retención de talento cualificado, reducción de tasas de absentismo y conflictividad laboral, entre otros. En cuanto a los trabajadores: aumento de su motivación, creatividad, confianza y compromiso con la empresa, mejora de su bienestar personal, satisfacción profesional y, en consecuencia, estabilidad laboral, fomento de la igualdad y corresponsabilidad entre hombres y mujeres, reducción del estrés y mejora de la salud de los trabajadores. [5] [9] [19]

A pesar de que la mayor parte de estas medidas no tienen ningún coste o este es mínimo, su implementación en las empresas españolas es todavía escasa [1]. La aplicación obligatoria por ley de estas [15] [17] está solo limitada a algunos conceptos generales y, por tanto, la voluntariedad en el resto de conceptos quedará relegada a la política que decida seguir cada empresa.

Las medidas de conciliación personal, familiar y laboral aplicables al sector comercial detallista español son: 
- Medidas establecidas por ley (de cumplimiento obligatorio) [15]: permiso de maternidad, permiso de paternidad, permiso de lactancia, medidas de seguridad durante embarazo y lactancia, excedencia voluntaria y excedencia por cuidado de hijos y otros familiares, reducción o modificación de la jornada laboral, permiso individual por formación del trabajador (aplicables a microempresas y grandes empresas).

- Medidas corporativas (de cumplimiento opcional) [7] [18]: flexibilidad horaria, jornada intensiva, teletrabajo, teletrabajo parcial, trabajo compartido, organización de turnos, responsabilidad en tiempo de reuniones, reducción de la pausa para comer, sustitución de horas extras por días libres, banco de horas, semana laboral comprimida, reducción personalizada de la jornada, vacaciones fraccionadas a la carta, días de permiso por horas a cuenta, asesoramiento personal, formación de los trabajadores, mes sabático por años de antigüedad, compra de días de vacaciones (aplicables a microempresas y grandes empresas), y plan luces apagadas, espacio de guardería, gimnasio, economato, comedor, pago adicional para guarderías, ayudas para los estudios de los hijos, ayudas por discapacidad, ayudas por hijos con discapacidad, transporte hasta recinto laboral (aplicables solo a grandes empresas).

\section{Recomendaciones: medidas de conciliación más adecuadas para el sector}

El sector comercial detallista español, por su tipología y en términos de conciliación, presenta una serie de necesidades específicas:

- Horario de apertura extenso: el sistema de venta tradicional, mayoritario en el sector, requiere de personal para cubrir este tiempo.

- Retención de capital humano: los trabajadores de cara al público son uno de los activos más valiosos de los retailers físicos y, por tanto, se debe retener y evitar la rotación.

- Necesidades diferentes según la empresa: según el tamaño de la empresa (microempresas y grandes empresas, mayoritarias en el sector) les necesidades serán diferentes.

Los factores tiempo y espacio son clave en el momento de impulsar una conciliación real. En este sentido, el desarrollo tecnológico contribuirá a unir ambos conceptos. Trabajar de una manera más digital, a través de nuevos programas informáticos y nuevos dispositivos, permitirá hacerlo desde espacios diferentes a la propia oficina (trabajo remoto). Este sistema requiere del trabajo por objetivos y con unos plazos establecidos para conseguir resultados exitosos.

Con el objetivo de concluir el presente estudio, se presenta a continuación una serie de recomendaciones para la implementación de las medidas de conciliación más adecuadas a este sector:

- A nivel empresarial:

Fomentar la igualdad y la paridad entre los trabajadores, fomentar la motivación y satisfacción de los mismos, revisar el actual sistema contractual aplicable, promover y facilitar el uso y disfrute de medidas de conciliación, especialmente las relativas a la gestión horaria, implementar un software para la planificación inteligente de los horarios, el servicio de autobús e impulsar cualesquiera otras medidas de conciliación familiar, de salud y profesional que sean necesarias para aumentar la satisfacción y motivación de los trabajadores. Estas acciones se deberán implementar siempre con el apoyo del nivel directivo y desde la integración en el ADN de la empresa.

- A nivel gubernamental e institucional:

Dotar una partida del presupuesto a impulsar políticas en pro de la conciliación personal, familiar y laboral en las empresas de este sector: para que hombres y mujeres tengan las mismas opciones laborales, para que los hijos/hijas de los trabajadores puedan ser criados por sus progenitores y para que las 
personas con necesidades especiales puedan ser atendidas por sus propios familiares. Para ponerlo en práctica será necesario que las instituciones apoyen la igualdad entre hombres y mujeres e impulsen un cambio social y una reorganización del tiempo y espacio dedicados al trabajo [12]. Del mismo modo, será recomendable: impulsar la actualización de la legislación vigente y la creación de la legislación necesaria para cubrir todos los aspectos relevantes en materia de conciliación, y trabajar para supervisar y controlar una implementación real y no manipulada de las medidas de conciliación.

\section{Bibliografía}

1. CHINCHILLA, N. et al. (2006). «Análisis sectorial de las políticas de conciliación». Conclusiones del estudio IFREI 2006 basado en 360 empresas [en línea]. https://media.iese.edu/research/pdfs/ESTUDIO-41.pdf.

2. Comercionista.com (2017). «¿Qué tipos de contratos laborales existen?» [en línea]. https://www.comercionista.com/personal/tipos-de-contratos-laborales/

3. Comunicarseweb (2014). «Segundo Estudio sobre Situación de la Conciliación en España» [en línea]. http:// www.comunicarseweb.com.ar/biblioteca/segundo-estudio-sobre-situacion-de-la-conciliacion-en-espana.

4. Confederación Española de Comercio (2016). «El comercio en cifras. Balance anual 2016» [en línea]. http:// www.cec-comercio.com/wp-content/uploads/2014/04/El-comercio-en-cifras_Balance-2016.pdf.

5. Cuentafacto.es (2018). «Conciliación vida laboral y familiar en 2018: ventajas y medidas a tener en cuenta» [en línea]. http://www.cuentafacto.es/tu-interes/conciliacion-vida-laboral-familiar-2018-ventajas-medidas-tenercuenta/.

6. Del Barrio, A. (2018). «Los horarios de trabajo inteligentes incrementan la productividad y las ventas» [en línea]. https://madridretailcongress.com/los-horarios-de-trabajo-inteligentes-incrementan-la-productividad-ylas-ventas/

7. Equipos\&Talento (2017). «10 medidas para aumentar la productividad y mejorar la conciliación laboral» [en línea]. https://www.equiposytalento.com/noticias/2017/05/02/10-medidas-para-aumentar-la-productividad-y-mejorar-la-conciliacion-laboral/.

8. Eurofound (2012). «Working conditions in the retail sector» [en línea]. https://www.eurofound.europa.eu/sites/ default/files/ef_files/docs/ewco/tn1109058s/tn1109058s.pdf.

9. Fundación Adecco (2015). «Por qué la conciliación beneficia a las empresas» [en línea]. https://fundacionadecco.org/azimut/por-que-la-conciliacion-beneficia-a-las-empresas/

10. Fundación FADE (2014). «Estudio sobre conciliación y familia» [en línea]. https://www.fundacionfade.org/images/informe_final_estudio_foro_debate_social_2014.pdf.

11. Fundación MásFamilia (2018). «¿Cuánto cuestan las medidas de conciliación?» [en línea]. https://www.masfamilia.org/blog/conciliacion/cuanto-cuestan-las-medidas-de-conciliacion.

12. Gobierno Vasco (2011). «¿Qué es la conciliación personal, familiar y laboral?» \{en línea]. http://www.euskadi. eus/que-es-la-conciliacion-personal-familiar-y-laboral/web01-a2concil/es/.

13. INE. Instituto Nacional de Estadística [en línea]. https://www.ine.es/.

14. Infoautónomos (2018). «El sector retail en España» [en línea]. https://infoautonomos.eleconomista.es/crecimiento-empresarial/sector-retail/.

15. Ley 39/1999, de 5 de noviembre, para promover la conciliación de la vida familiar y laboral de las persones trabajadoras [en línea]. http://noticias.juridicas.com/base_datos/Laboral//39-1999.html.

16. Ley 1/2004, de 21 de diciembre, de Horarios Comerciales [en línea]. http://noticias.juridicas.com/base_datos/ Privado/l1-2004.html.

17. Ley $3 / 2007$, de 22 de marzo, para la igualdad efectiva de mujeres y hombres [en línea]. http://noticias.juridicas.com/base_datos/Admin/lo3-2007.html.

18. People-first (2018). «La conciliación aumenta la productividad y el bienestar» [en línea]. https://www.peoplefirst.blog/la-conciliacion-aumenta-la-productividad-y-el-bienestar/ 
19. Prevenblog.com (2016). «Claves para implantar un plan de conciliación en una organización» [en línea]. http:// prevenblog.com/claves-para-implantar-un-plan-de-conciliacion-en-una-organizacion/.

20. RAE. Real Academia Española [en línea]. http://www.rae.es/.

21. VARGAS, M. (2004). «La conciliación de la vida laboral y familiar» [en línea]. http://www.mujeresenred.net/ IMG/pdf/conciliacion_lab.pdf.

Cita recomendada: MORALES, Carolina. Medidas para conciliar en el sector retail. Recomendaciones para la implementación de medidas de conciliación en el sector comercial detallista español. Oikonomics [en línea]. Mayo 2020, n. 13, pp. 1-7. ISSN: 2339-9546. DOI: https://doi. org/10.7238/o.n13.2009

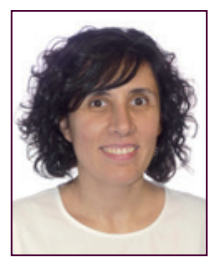

\section{Carolina Morales Pallarés}

cmoralespal@uoc.edu

Graduada en Administración y Dirección de Empresas por la UOC.

Máster en Dirección de Marketing por EAE.

Especialista en retail marketing.

Especialista en retail marketing con más de 15 años de experiencia, ha liderado proyectos en empresas de distinto tamaño y sector tanto a nivel nacional como internacional. Su área de conocimiento y especialidad es el marketing aplicado al sector del comercio detallista.

Sus intereses de investigación se centran en temas de género, conciliación laboral e innovación empresarial. Ganadora del mejor TFG de los Estudios de Economía y Empresa con perspectiva de género del curso 2018/19.

Los textos publicados en esta revista están sujetos -si no se indica lo contrario- a una licencia de Reconocimiento 4.0 Internacional de Creative Commons. Puede copiarlos, distribuirlos, comunicarlos públicamente, hacer obras derivadas siempre que reconozca los créditos de las obras (autoría, nombre de la revista, institución editora) de la manera especificada por los autores o por la revista. La licencia completa se puede consultar en https://creativecommons.org/licenses/by/4.0/deed.es_ES.

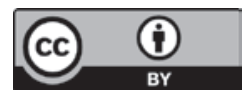

